\title{
Mechanisms and Consequences of Chromosomal Translocation
}

\author{
Charles S. Rabkin ${ }^{1}$ and Siegfried Janz ${ }^{2}$ \\ 1 Viral Epidemiology Branch, Division of Cancer Epidemiology and Genetics, National Cancer Institute, \\ Bethesda, MD 20892
}

2 Department of Pathology, University of Iowa Roy J. and Lucille A. Carver College of Medicine, Iowa City, IA 52242

\begin{abstract}
A 2006 National Cancer Institute workshop on chromosomal translocations brought together laboratory, clinical, and population scientists to cross-fertilize and catalyze research on this important disease process. The deliberations revealed significant contrasts between two types of translocations that result in either deregulated expression of oncogenes or formation of novel fusion genes. The oncogene-activating MYC-IGH exchange is the best-elucidated translocation in terms of molecular structure and functional consequences and the anti-apoptotic effects of constitutively activated BCL2 facilitate the detection of the $B C L 2-I G H$ translocation in blood as a potential predictor of cancer risk. In comparison, the fusion-gene translocations such as BCR-ABL and the varied recombinations of MLL have well-described clinical manifestations but are less well-defined with regard to their mechanisms of generation. Interdisciplinary collaboration on chromosomal translocations should yield additional insights regarding their biologic significance and potential as targets for intervention.
\end{abstract}

\section{Keywords}

chromosome; translocation; oncogene; fusion-gene; meeting report

\begin{abstract}
A National Cancer Institute workshop on chromosomal translocations in cancer was held in October 2006 at the Airlie Conference Center, Warrenton, Virginia. The workshop brought together laboratory, clinical, and population scientists to cross-fertilize and catalyze relevant research. The proceedings papers highlight recent findings on the molecular mechanisms of chromosomal translocations ${ }^{1}$. A particular focus is the MYC-IGH exchange, arguably the bestelucidated translocation in terms of chromosomal and molecular structure, DNA repair and cellular stress-response signaling pathways, and modeling in transgenic mice. Epidemiologic understanding of oncogene-activating translocations primarily derives from unique features of $B C L 2$, which, when expressed by juxtaposition to $I G H$ enhancers, promotes survival and expansion of affected cells that are readily sampled in peripheral blood. By comparison, the fusion-gene translocations have well-described clinical consequences but only recently have been modeled in mice, and their mechanisms of generation are still unclear. Translocations and other chromosomal aberrations are potentially valuable predictors of human cancer risk. An historical perspective and considerations of heritable translocations in mice round out a comprehensive overview of this important disease process ${ }^{1}$.
\end{abstract}

Correspondence and reprint requests to Charles Rabkin or Siegfried Janz: Charles S. Rabkin, M.D., M.Sc., Senior Investigator, Viral Epidemiology Branch, DCEG, National Cancer Institute, 6120 Executive Blvd., EPS/7082, Bethesda, MD 20892, E-MAIL: rabkinc@mail.nih.gov, Siegfried Janz, M.D., Professor, Department of Pathology, CCOM, The University of Iowa, 200 Hawkins Drive, Iowa City, IA 52242, E-MAIL: siegfried-janz@uiowa.edu. 
These wide-ranging observations reveal several significant contrasts between two types of translocations that result in either deregulated expression of oncogenes or formation of novel fusion genes (Table). First, the biologic mechanisms underlying oncogene-activating translocations appear to represent accidents of physiologic DNA recombination and modification processes of lymphocyte development and the adaptive immune response. The potential mechanisms of fusion-gene translocations are more diverse and speculative. Epidemiologic evidence in infant leukemia implicates defective balance of metabolic pathways, which suggests exciting opportunities for translocation prevention in the future. However, there is need for caution in generalizing observations from this or any other individual model system, as circumstantial evidence indicates that different mechanisms operate even within the same tumor type. A case in point is the $M Y C$-activating translocation in Burkitt lymphoma, for which molecular structure differs depending upon the epidemiologic setting. Beyond the general relation to the immune response, it cannot be assumed that mechanisms determined for translocations in sporadic or immunodeficiency-associated Burkitt lymphoma are also operative in endemic disease. Epidemiologic studies need to retain a broad perspective, free of assumptions, when considering biologic evidence for causation.

Second, since proto-oncogenes are frequently involved in controlling cellular growth, proliferation and/or programmed death, aberrant oncogene expression from chromosomal translocation frequently leads to cellular immortalization and clonal expansion. In contrast, genes prone to in-frame fusion with partner loci on other chromosomes often encode transcription factors regulating differentiation at the cellular, tissue and organismal level; consequently, fusion-gene translocations commonly lead to arrested or aberrant development. These distinctions are not absolute and important exceptions include translocations associated with T-ALL, CML, mantle cell lymphoma, and some rare forms of sarcoma. However, at the risk of oversimplifying complex biology, it can be said that the pathologies associated with oncogene-activation tend to be limited and defined, whereas the pathologies associated with fusion genes may be broad and less predictable. Fusion-gene translocations are also more difficult than oncogene translocations to recapitulate in model systems, such as transgenic mice.

Third, while oncogene-activating translocations are tightly associated with lymphoid malignancies and fusion-gene translocations are classically recognized as hallmarks of hematopoietic neoplasms and sarcomas, there is increasing evidence for involvement of both types of translocations in the pathogenesis of the more common carcinomas. Although not discussed in the present monograph, instructive examples include the fusion-gene PAX8$P P A R G$ in follicular thyroid carcinoma and the oncogene-activating TMPRSS2-ERG in prostate cancer. The latter finding is particularly significant in that ERG is also involved in fusion genes of Ewing's sarcoma, indicating that this ETS transcription factor may be targeted by both types of translocations. Based on the ever-increasing resolution of molecular cytogenetic methods, it is likely that additional solid tumors will be revealed to harbor recurrent translocations, obscured to date in the notoriously complex karyotypes of these neoplasms. Intriguingly, some oncogene translocations, particularly $\mathrm{t}(14 ; 18)(\mathrm{q} 32 ; \mathrm{q} 21)$, occur in most adults without apparent adverse effects even at the cellular level. On the other hand, fusiongene translocations are generally considered to signal quick progression to frank disease, although some specific types such as BCR-ABL, TEL-AML1, AML1-ETO and E2A-PBX1, may be detected in healthy individuals of all ages. These observations point to poorly understood differences in the oncogenic potency of oncogene vs. fusion translocations, while accommodating the well-established reality that both types of translocation must be complemented by collaborating tumor progression events before complete neoplastic transformation occurs. 
Fourth, there are disparities within particular model systems of basic vs. epidemiologic knowledge. For example, t $(8 ; 14)(\mathrm{q} 24 ; \mathrm{q} 32)$ is a molecularly defined abnormality in an exhaustively studied genetic system including a well-developed mouse analogue, but has relatively little epidemiologic characterization. Conversely, there is a wealth of information regarding the epidemiology of $M L L$ abnormalities and their relationship to disease, but more limited understanding of the biologic mechanisms of MLL-driven neoplasia, apart from MLL's histone methyltransferase activity and ability to activate Hox genes. The mouse models parallel this distinction: there is a long and informative history of transgenic oncogene models, while sophisticated gene-targeting approaches have just recently begun to provide insights into the role of fusion-genes in neoplastic cell development. Future research using mouse models of cancer-associated translocations could help elucidate the epidemiologic variation in their oncogenic potency and thus close the gap in our understanding of basic vs. epidemiologic aspects of oncogenic translocations.

Last, the two types of translocations have differing implications for translational research aimed at developing new therapeutic and preventive approaches to cancer. Given current technology, protein kinases commonly deregulated by fusion genes are more readily targeted by small-drug inhibitors than transcription factors commonly encoded by oncogenes. The potential of the former approach is illustrated by the $A B L$ tyrosine kinase inhibitor, imatinib, which is now exceeding expectations with clinical utility in disorders lacking the $t(9 ; 22)$ (q34;q11) $B C R-A B L$ fusion gene. Drugs like imatinib often target proteins that are altered by conformational or other changes induced by gene fusion. In contrast, the challenges of targeting transcription factors, regardless if they are deregulated by oncogene-activating or fusion gene translocations, are significantly greater and will require mounting multiple technological hurdles. Although not yet yielding clinically significant mitigation, efforts are underway to control deregulated oncogenes using iRNA, monoclonal antibody, or small drug inhibitors. The development of imatinib was based on more than two decades of multidisciplinary research following the discovery of the Philadelphia chromosome, the cytogenetic indication of $t(9 ; 22)$ (q34;q11). Further collaboration of laboratory, clinical, and population scientists studying chromosomal translocations is bound to generate additional insights regarding their biologic significance and potential as targets for intervention.

\section{Acknowledgements}

The authors thank Dr. Frederic Barr and two anonymous reviewers for providing helpful suggestions for this manuscript.

\section{References}

1. Rabkin, CS.; Janz, S., editors. Mechanisms and consequences of chromosomal translocations. J Natl Cancer Inst Monogr; Proceedings of a National Cancer Institute Workshop; 2006 October 22-24; Warrenton, Virginia. 2008. In press 
Table

Distinguishing features of chromosomal translocations that activate cellular oncogenes or generate novel fusion genes

\begin{tabular}{lll}
\hline & Oncogene-activating & Fusion gene-generating \\
\cline { 2 - 3 } Translocation mechanism: & adaptive immunity machinery & metabolic imbalance \\
Normal gene function: & growth, proliferation, apoptosis & development and differentiation \\
Pathophysiology: & immortalization, clonal expansion & arrested/deranged development \\
Occurrence in neoplasms: & lymphoid & hematologic, sarcomas, carcinomas \\
Occurrence in healthy individuals: & common and tolerated & and may progress quickly \\
Human characterization: & biologic $>$ epidemiologic & gene-targeted \\
Mouse models: & conventional transgenic & proven \\
Therapeutic target: & difficult & rebalance metabolic pathways? \\
Prevention strategy: & immune modulation & \\
\end{tabular}

